\title{
Generalized stationary processes of Markovian character
}

by

\author{
K. URBANIK (Wroclaw)
}

\section{Introduction}

The theory of linear prediction developed by Kolmogorov [7] and Wiener [15] treats the problem of extrapolating from the known past of some stochastic process to its future. The purpose of this note is a study of generalized stationary processes for which the prediction of the future in terms of the past up to time $t$ depends only upon the behaviour of the process in an arbitrarily small neighbourhood of $t$. For ordinary Gaussian processes a similar problem was investigated by Lévy [9], who established the canonical form of processes for which random functions $x_{1}(t), x_{2}(t)$, $\ldots, x_{n}(t)$ may be found such that if they are known, then the behaviour of the process in $(t, \infty)$ is independent of all other informations on the values of this process in $(-\infty, t)$.

Let $\mathscr{D}$ denote the space of all infinitely differentiable complex-valued functions defined on the real line whose supports are compact. The support of a function $\varphi$ from $\mathscr{Q}$, i. e. the closure of the set $\{t: \varphi(t)$ $\neq 0\}$, will be denoted by $s(\varphi)$. In the sequel for every real number $h$ we shall use the notation $\left(\tau_{h} \varphi\right)(t)=\varphi(t+h)$. A sequence $\varphi_{1}, \varphi_{2}, \ldots$ from $\mathscr{D}$ is said to be $\mathscr{D}$-convergent to $\varphi$ if the set $\bigcup_{n=1}^{\infty} s\left(\varphi_{n}\right)$ is bounded and for every $k(k \geqslant 0)$ the sequence of derivatives $\varphi_{1}^{n=1}, \varphi_{2}^{(k)}, \ldots$ uniformly converges to $\phi^{(k)}$. The space $\mathscr{D}^{\prime}$ of Schwartz distributions is the space of all continuous linear functionals on $\mathscr{D}([13]$, p. 24).

Let us consider the totality of complex-valued random rariables with mean 0 and finite variance which constitute a Hilbert space $\mathfrak{S}$ with inner product $(\xi, \eta)=E(\xi \cdot \bar{\eta})$, where $E(\xi)$ denotes the expectation of the random variable $\zeta$. A continuous linear mapping $X$ from $\mathscr{D}$ into $\mathfrak{G}$ is called a generalized stochastic process. This notion is due to Itô $([5]$, p. 210). Ordinary stochastic processes $x(t)$ continuous in quadratic mean may be treated as generalized ones. Namely, we make the generalized process $X(\varphi)=\int_{-\infty}^{\infty} x(t) \varphi(t) d t$ to correspond to the process $x(t)$. 
A generalized stochastic process $X$ is said to be stationary if for every real number $h$ we have the equality $\left(X\left(\tau_{h} \varphi\right), X\left(\tau_{h} \psi\right)\right)=(X(\varphi), X(\psi))$ $(\varphi, \psi \in \mathscr{D})$. Stationary processes which are ordinary in the wide sense (see [2], p.512) are stationary as generalized processes. Conversely, every ordinary process which is stationary as a generalized one is also stationary in the wide sense as an ordinary process.

Itô [5.] has developed the spectral theory of generalized stationary processes. In particular, he has proved that for every generalized stationary process $X$ there exists a measure $\mu_{X}$ defined on the class of all Borel subsets of the real line such that for an integer $s$

and

$$
\int_{-\infty}^{\infty}(1+|t|)^{-s} \mu_{X}(d t)<\infty
$$

$$
(X(\varphi), X(\psi))=\int_{-\infty}^{\infty} \hat{\varphi}(t) \hat{\psi}(t) \mu_{X}(d t),
$$

where $\hat{\varphi}$ denotes the Fourier transform of $\varphi$, i. e.

$$
\hat{\varphi}(t)=\int_{-\infty}^{\infty} e^{i t u} \varphi(u) d u .
$$

The measure $\mu_{X}$ is uniquely determined and is called the spectral measure of the process $X$. The positive definite Schwartz distribution

$$
R_{X}(\varphi)=\int_{-\infty}^{\infty} \hat{\varphi}(t) \mu_{X}(d t)
$$

is called the covariance distribution of the process $X$. The relationship between the inner product and the covariance distribution is given by the formula

$$
(X(\varphi), X(\psi))=R_{X}(\varphi * \tilde{\psi})
$$

where * denotes the convolution and $\tilde{\psi}(t)=\overline{\psi(-t)}$. We remark that a generalized stationary process is an ordinary one if and only if its spectral measure is finite on the whole line. Moreover, if $X(\varphi)=\int_{-\infty}^{\infty} x(t) \varphi(t) d t$, where $x(t)$ is an ordinary process, then

$$
R_{X}(\varphi)=\int_{-\infty}^{\infty} r_{x}(t) \varphi(t) d t
$$

where $r_{x}(t)$ is the covariance function of $x(t): r_{x}(t)=\int_{-\infty}^{\infty} e^{i t u} \mu_{X}(d u)$.

Let $A$ be a subset of $\mathfrak{H}$. By $[A]$ we shall denote the least subspace of $\mathfrak{G}$ containing the set $A$. For every generalized process $X$ we can define a corresponding subspace of $\mathfrak{S}$ as follows:

$$
\mathfrak{H}_{X}=[\{X(\varphi): \varphi \in \mathscr{F}\}] .
$$

The Hilbert space $\mathfrak{S}_{X}$ is isomorphic to the space $L^{2}\left(\mu_{X}\right)$ of all complex- valued functions which are square-integrable with respect to the spectral measure $\mu_{X}$. A natural isomorphism $\iota: \mathfrak{G}_{X} \rightarrow L^{2}\left(\mu_{X}\right)$ is the extension of the mapping $\iota(X(\varphi))=\hat{\varphi}$. If $X$ is an ordinary process, then we have the equality $\iota(x(t))(u)=e^{i t u}$.

For any set $I$ we put

$$
\mathfrak{S}_{X}^{I}=[\{X(\varphi): \varphi \in \mathscr{D}, s(\varphi) \subset I\}] .
$$

The linear least squares prediction of $X(\varphi)$, based on the full past of the process up to time $t_{0}$, is the orthogonal projection of $X(\varphi)$ into the subspace $\mathfrak{S}_{X}^{\left(-\infty, t_{0}\right)}$. In the sequel this prediction will be denoted by $\pi_{t_{0}} X(\varphi)$.

A generalized process $X$ will be called deterministic if, for every $t$,

$$
\mathfrak{S}_{\left.X^{-\infty}, t\right)}=\mathfrak{H}_{X}
$$

and completely non-deterministic if

$$
\bigcap_{-\infty<i<\infty} \mathfrak{H}_{X}^{(-\infty, t)}=\{0\} \neq \mathfrak{H}_{\mathbf{X}} .
$$

Every process is the sum of two orthogonal processes, one deterministic and the other completely non-deterministic. Rozanov [12] extended the famous Kolmogorov-Krein criterion ([7], [8]) onto the class of generalized processes. Namely, he proved that a generalized stationary process $X$ is deterministic if and only if

$$
\int_{-\infty}^{\infty} \frac{\log g_{X}(t)}{1+t^{2}} d t=-\infty,
$$

where $g_{X}$ is the density function of the absolutely continuous component of the spectral measure $\mu_{X}$. Further, the process $X$ is completely non-deterministic if and only if its spectral measure is absolutely continuous and

$$
\int_{-\infty}^{\infty} \frac{\log g_{X}(t)}{1+t^{2}} d t>-\infty
$$

\section{Markovian character}

A generalized stationary process $X$ is said to be of Markovian character if for every $t_{0}$

$$
\pi_{t_{0}} X(\varphi) \in \bigcap_{u<t_{0}} \mathfrak{S}_{X}^{\left(u, t_{0}\right)}
$$


whenever $s(\varphi) \subset\left(t_{0}, \infty\right)$. For such a process the prediction of the future based on the full past up to time $t_{0}$ depends only upon the behaviour of the process in an arbitrarily small neighbourhood of $t_{0}$. From stationariness we infer that $X$ is of Markovian character if and only if

$$
\pi_{0} X(\varphi) \in \bigcap_{u<0} \mathfrak{H}_{X}^{(u, 0)}
$$

whenever the support of $\varphi$ is contained in the right half-line. Moreover, an ordinary stationary process $x(t)$ is of Markovian character if and only if for every positive $t$ we have the relation

$$
\pi_{0} x(t) \in \bigcap_{u<0} \mathfrak{S}_{X}^{(u, 0)}
$$

Now we shall quote simple examples of deterministic as well as completely non-deterministic processes of Markovian character. In the sequel by $x^{(n)}(t)$ we shall denote the $n$-th derivative in quadratic mean of the process $x(t)$.

1. Random oscillations. Let us consider a system $\xi_{1}, \xi_{2}, \ldots, \xi_{n}$ of orthogonal random variables with mean 0 and finite variance and a system $\lambda_{1}, \lambda_{2}, \ldots, \lambda_{n}$ of real numbers different from one another. Setting

$$
x(t)=\sum_{k=1}^{n} \xi_{k} \exp \left(i \lambda_{k} t\right)
$$

we get a stationary process with the covariance function

$$
r_{x}(t)=\sum_{k=1}^{n} b_{k} \exp \left(i \lambda_{k} t\right),
$$

where the coefficients $b_{1}, b_{2}, \ldots, b_{n}$, being the variances of $\xi_{1}, \xi_{2}, \ldots, \xi_{n}$ respectively, are non-negative. Every stationary process having such a covariance function is equivalent to the sum (1) and will be called a random oscillation. It is very easy to prove the equality

$$
\pi_{0} x(t)=\sum_{k=1}^{n} \frac{\Delta_{k}}{\Delta} \exp \left(i \lambda_{l_{k}} t\right),
$$

where

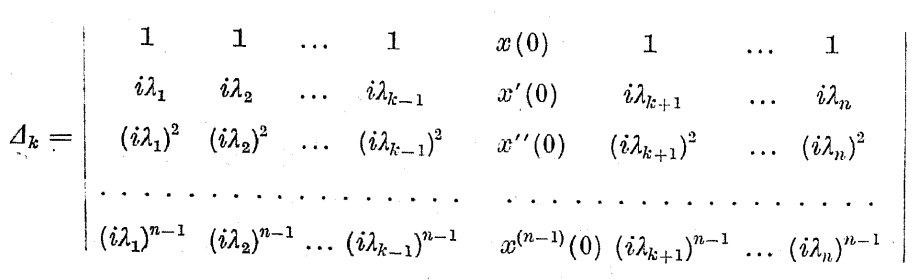

and $\Delta$ is the Vandermonde determinant of $i \lambda_{1}, i \lambda_{2}, \ldots, i \lambda_{n}$. Thus the prediction $\pi_{0} x(t)$ is a linear combination of derivatives $x(0), x^{\prime}(0)$, $\ldots, x^{(n-1)}(0)$ and consequently belongs to $\bigcap_{u<0} \mathfrak{S}_{x}^{(u, 0)}$, whence the Markovian character of $x(t)$ follows. On the other hand, by simple computations we get the equality $\pi_{0} x(t)=x(t)$. Hence we infer that the process $a(t)$ is deterministic.

2. Analytic processes. An ordinary stationary process $x(t)$ is called analytic if it is infinitely differentiable and can be expanded in the power series

$$
\sum_{n=0}^{\infty} \frac{x^{(n)}(0)}{n !} t^{n}
$$

convergent in quadratic mean. For instance, all random oscillations are analytic processes. By Loève's theorem ([11], p. 471 ), a stochastic process is analytic if and only if its covariance function can be extended to an entire function on the complex plane. Moreorer, if the process and all its derivatives are separable, then almost all its sample functions can be extended to entire functions on the complex plane ([1], p. 438). The prediction of analytic processes can be expressed in terms of derivatives $x(0), x^{\prime}(0), \ldots$ as follows:

$$
\pi_{0} x(t)=\sum_{n=0}^{\infty} \frac{x^{(n)}(0)}{n !} t^{n} .
$$

Since all the derivatives $x(0), x^{\prime}(0), \ldots$ belong to $\bigcap_{u<0} \mathfrak{S}_{x}^{(u, 0)}$, analytic processes are of Markovian character. Moreover, $\pi_{0} x(t)=x(t)$ and, consequently, they are deterministic.

3. Generalized processes with orthogonal values. We say, following Gelfand [3], that a generalized stationary process $X$ has orthogonal values if for every pair $\varphi, \psi$ from $\mathscr{Z}$ satisfying the condition $s(\varphi) \cap s(\psi)$ $=0$ the equality $(X(\varphi), X(\psi))=0$ holds. The covariance distribution of processes with orthogonal values is a linear combination of the Dirac $\delta$-distribution and its derivatives (see [3]). If the process is not identically equal to 0 , then its spectral measure is absolutely continuous and the spectral density, i. e. the first derivative of the spectral measure is a polynomial. Of course, every such process is completely non-deterministic. For every function $\varphi$ with $s(\varphi) \subset(0, \infty)$ we have the equality $\pi_{0} X(\varphi)=0$, which implies the Markovian character of processes with orthogonal values.

4. Processes whose spectral density is the reciprocal of a polynomial. Let us consider an ordinary stationary process $x(t)$ with 
absolutely continuous spectral measure having the spectral density of the form

$$
g_{x}(t)=\frac{1}{W(t)},
$$

where $W(t)$ is a polynomial. Of course, the integral

$$
\int_{-\infty}^{\infty} \frac{\log g_{x}(t)}{1+t^{2}} d t
$$

is finite and, consequently, the process $x(t)$ is completely non-deterministic. Moreover, an explicit formula for the prediction is well known (see [6], p. 127):

$$
\pi_{0} x(t)=\sum_{k=0}^{n} c_{k}(t) x^{(k)}(0) \quad(t>0)
$$

where $c_{0}(t), c_{1}(t), \ldots, c_{n}(t)$ are non-random coefficients. Since the linear combination of derivatives $x(0), x^{\prime}(0), \ldots, x^{(n)}(0)$ belongs to $\bigcap_{u<0} \mathfrak{F}_{x}^{(u, 0)}$, all processes with the spectral density of form (3) are of Markovian character.

Stationary processes $x(t)$ called Markov in the wide sense (see for the definition [2], p. 233) belong to the class under consideration provided they are not constant. Their spectral density is given by the formula

$$
g_{x}(t)=\frac{a}{|2 \pi i t+b|^{2}}
$$

where $a$ is a positive number and $b$ is a complex number with positive real part. Moreover, the covariance function $r_{x}(t)$ is equal to $r_{x}(0) e^{-b t}$ $(t>0)$. They are the only non-constant stationary processes for which the prediction is of the form $\pi_{0} x(t)=C(t) x(0)(t>0)$ (see [2], p. 233).

5. A completely non-deterministic process for which the prediction depends upon infinitely many derivatives. Let $x(t)$ be a stationary process with absolutely continuous spectral measure having the spectral density

$$
g_{x}(t)=\frac{c}{\prod_{k=1}^{\infty}\left(1+t^{2} / a_{k}^{2}\right)}
$$

where $c, a_{1}, a_{2}, \ldots$ are positive numbers,

$$
a_{1}<a_{2}<\ldots
$$

and

$$
\varlimsup_{k \rightarrow \infty} \frac{a_{k}}{a_{k+1}}<1 .
$$

By simple computations we get the inequality

$$
\int_{-\infty}^{\infty} \frac{\log g_{x}(t)}{1+t^{2}} d t=\pi \log \frac{c}{\prod_{k=1}^{\infty}\left(1+1 / a_{k}\right)^{2}}>-\infty .
$$

Thus, by the Kolmogorov-Krein criterion, the process $x(t)$ is completely non-deterministic. We shall now prove that the process $x(t)$ is of Markovian character. From (6) it follows that there exist an integer $k_{0}$ and a positive number $b$ smaller than 1 such that the inequality $a_{k} / a_{n} \leqslant b^{n-k}$ holds whenever $n>k \geqslant k_{0}$. Taking into account this inequality, we get the following one for $k>k_{0}$ :

$$
\begin{aligned}
& \prod_{\substack{n=1 \\
n \neq k}}^{\infty} 1-\frac{a_{k}}{a_{n}} \mid=\prod_{n=1}^{k_{0}-1}\left(\frac{a_{k}}{a_{n}}-1\right) \prod_{n=k_{0}}^{k-1}\left(\frac{a_{k}}{a_{n}}-1\right) \prod_{n=k+1}^{\infty}\left(1-\frac{a_{k}}{a_{n}}\right) \\
\geqslant & \prod_{n=1}^{k_{0}-1}\left(\frac{a_{k}}{a_{n}}-1\right) \prod_{n=k_{0}}^{k-1}\left(b^{n-k}-1\right) \prod_{n=1}^{\infty}\left(1-b^{n}\right) \geqslant \prod_{n=1}^{k_{0}-1}\left(\frac{a_{k}}{a_{n}}-1\right) \prod_{n=1}^{\infty}\left(1-b^{n}\right)^{2} .
\end{aligned}
$$

Hence and from (5) we obtain the inequality

$$
A=\inf _{\substack { 1 \leqslant k<\infty \\
\begin{subarray}{c}{n=1 \\
n \neq k{ 1 \leqslant k < \infty \\
\begin{subarray} { c } { n = 1 \\
n \neq k } }\end{subarray}}^{\infty}\left|1-\frac{a_{k}}{a_{n}}\right|>0 .
$$

Let us introduce an auxiliary function of the complex variable

$$
F(z)=\prod_{n=1}^{\infty}\left(1+\frac{i z}{a_{n}}\right)
$$

This function is an entire function of exponential type. Since

$$
F^{\prime}\left(i a_{k}\right)=\frac{i}{a_{k}} \prod_{\substack{n=1 \\ n \neq k}}^{\infty}\left(1-\frac{a_{k}}{a_{n}}\right)
$$

we have, in view of (7),

$$
A=\inf _{1 \leqslant k<\infty}\left|a_{k} F^{\prime}\left(i a_{k}\right)\right|>0 .
$$


Given a positive number $t$, we put

$$
\begin{gathered}
w(z)=\frac{|F(z)|}{A} \sum_{k=1}^{\infty} \frac{e^{-t a_{k}}}{\left|1+i z / a_{k}\right|}, \quad v(z)=\sum_{k=1}^{\infty} \frac{F(z) e^{-t a_{k}}}{F^{\prime}\left(i a_{k}\right)\left(z-i a_{k}\right)}, \\
v_{m}(z)=\sum_{k=1}^{m} \frac{\prod_{n=1}^{m}\left(1-i z / a_{n}\right) e^{-t a_{k}}}{F^{\prime}\left(i a_{k}\right)\left(z-i a_{k}\right)}+\sum_{k=m+1}^{\infty} \frac{\prod_{n=1}^{m}\left(1-i z / a_{n}\right) e^{-t a_{k}}}{F^{\prime}\left(i a_{k}\right)}
\end{gathered}
$$

By (8), we have on the whole complex plane

$$
\begin{aligned}
|v(z)| \leqslant \sum_{k=1}^{\infty} \frac{e^{-t a_{k}}|F(z)|}{A|1+i z| a_{k} \mid}=w(z) & =\frac{1}{A} \sum_{k=1}^{\infty} e^{-t a_{k}} \prod_{\substack{n=1 \\
n \neq k}}^{\infty}\left|1+\frac{i z}{a_{n}}\right| \\
& \leqslant \frac{1}{A} \sum_{k=1}^{\infty} e^{-t a_{k}} \exp \left(\sum_{n=1}^{\infty} a_{n}|z|\right) .
\end{aligned}
$$

Thus $v(z)$ is an entire function of exponential type. Further, we have the inequality $\left|v_{m}(u)\right| \leqslant w(u)(m=1,2, \ldots)$ on the real line. The sequence $v_{1}(z), v_{2}(z), \ldots$ of polynomials converges to $v(z)$ uniformly in every compact set. We shall now prove that all the functions $v(z), v_{1}(z)$, $v_{2}(z), \ldots$, regarded on the real line, belong to the space $L^{2}\left(\mu_{x}\right)$ and the sequence $v_{1}(u), v_{2}(u), \ldots$ converges to $v(u)$ in the sense of $L^{2}\left(\mu_{x}\right)$, where $\mu_{x}$ is the spectral measure of the process in question. To prove this it is sufficient to show that the function $w(u)$ belongs to $L^{2}\left(\mu_{x}\right)$. But this statement follows from the Schwarz inequality. In fact,

whence, setting

$$
w^{2}(u) \leqslant \frac{|F(u)|^{2}}{A^{2}} \sum_{k=1}^{\infty} e^{-t a_{k}} \sum_{n=1}^{\infty} \frac{e^{-t a_{n}}}{1+u^{2} / a_{n}^{2}},
$$

we get

$$
B=\frac{1}{A^{2}} \sum_{k=1}^{\infty} e^{-t a_{k}}
$$

$$
\begin{aligned}
\int_{-\infty}^{\infty} w^{2}(u) \mu_{x}(d u) & \leqslant B \sum_{n=1}^{\infty} e^{-t a_{n}} \int_{-\infty}^{\infty} \frac{|F(u)|^{2}}{1+u^{2} / a_{n}^{2}} \mu_{x}(d u) \\
& =B c \sum_{n=1}^{\infty} e^{-t a_{n}} \int_{-\infty}^{\infty} \frac{d u}{1+u^{2} / a_{n}^{2}}=B c \pi \sum_{n=1}^{\infty} a_{n} e^{-t a_{n}}<\infty
\end{aligned}
$$

We have proved that the function $v(u)$ is the limit in the sense of $L^{2}\left(\mu_{x}\right)$-convergence of a sequence of polynomials. From the equality

$$
\iota\left(x^{(n)}(0)\right)=(i u)^{n} \quad(n=0,1, \ldots)
$$

where $\iota$ is the natural mapping from $H_{x}$ onto $L^{2}\left(\mu_{x}\right)$, it follows that the random variable $\iota^{-1}(v)$ is the limit in quadratic mean of a sequence of linear combinations of derivatives $x(0), x^{\prime}(0), \ldots$ Thus

$$
\iota^{-1}(v) \in \bigcap_{u<0} \mathfrak{S}_{x}^{(u, 0)} \text {. }
$$

To prove the equality

$$
\pi_{0} x(t)=\iota^{-1}(v) \quad(t>0)
$$

it suffices to show that the random variable $x(t)-\iota^{-1}(v)$ is orthogonal to all random variables $x(s)(s<0)$. In the language of $L^{2}\left(\mu_{x}\right)$ the last statement can be written as follows:

$$
\int_{-\infty}^{\infty}\left(e^{i t u}-v(u)\right) e^{-i s u} g_{x}(u) d u=0 \quad(s<0) .
$$

Now we proceed to the proof of (12). Since $v\left(i a_{k}\right)=e^{-t a_{k}}(k=1$, $2, \ldots)$ and the functions $v(z), F(z)$ and $e^{i t z}$ are entire of exponential type, the quotient $\left(e^{i t z}-v(z)\right) / F(z)$ is the same one (see [10], p. 37). Put

$$
F^{*}(z)=\prod_{n=1}^{\infty}\left(1-\frac{i z}{a_{n}}\right) \text {. }
$$

For all complex numbers $z$ in the upper half-plane we have

$$
\left|F^{*}(z)\right| \geqslant \prod_{n=1}^{\infty}\left|\operatorname{Re}\left(1-\frac{i z}{a_{n}}\right)\right|=\prod_{n=1}^{\infty}\left(1+\frac{\operatorname{Im} z}{a_{n}}\right) \geqslant 1 .
$$

Hence it follows that the function $z^{2}\left(e^{i t z}-v(z)\right) e^{-i s z} / F(z) F^{*}(z) \quad(t>0$, $s<0)$ is analytic and of exponential type in the upper half-plane. Giren a complex number $z=\grave{\varrho} e^{i \theta}$, where $0 \leqslant \theta \leqslant \frac{1}{8} \pi$ or $\frac{7}{8} \pi \leqslant \theta \leqslant \pi$, for erery index $n$ we have

$$
\left|1+\frac{i \approx}{a_{n}}\right|^{2}=1-\frac{2 \varrho \sin \theta}{a_{n}}+\frac{\varrho^{2}}{a_{n}^{2}} \geqslant 1-\frac{\varrho}{a_{n}}+\frac{\varrho^{2}}{a_{n}^{2}} \geqslant \frac{3}{4} .
$$

Hence and from (8) we get

$$
\begin{gathered}
\left|\frac{z^{2}\left(e^{i t z}-v(z)\right) e^{-i s z}}{F(z) F^{*}(z)}\right| \leqslant\left|\frac{z^{2} e^{i(t-s) z}}{\prod_{n=1}^{\infty}\left(1+z^{2} \mid a_{n}^{2}\right)}\right|+\left|\frac{z^{2} e^{-i s z}}{A F^{*}(z)}\right| \sum_{n=1}^{\infty} \frac{e^{-t a_{n}}}{|1+i z| a_{n} \mid} \\
\leqslant \frac{\varrho^{2} e^{(s-t) \varrho \sin \theta}}{\prod_{n=1}^{\infty}\left(1+\varrho^{2} \cos 2 \theta / a_{n}^{2}\right)}+\frac{2 \sum_{n=1}^{\infty} e^{-t a_{n}} \varrho^{2} e^{s e \sin \theta}}{\sqrt{3} A \prod_{n=1}^{\infty}\left(1+\frac{2 \varrho \sin \theta}{a_{n}}+\frac{\varrho^{2}}{a_{n}^{2}}\right)^{1 / 2}} .
\end{gathered}
$$


Since $\sin \theta \geqslant 0$ and $\cos 2 \theta>0$ whenever $0 \leqslant \theta<\frac{1}{8} \pi$ or $\frac{7}{8} \pi \leqslant \theta \leqslant \pi$, $t>0$ and $s<0$, the function $z^{2}\left(e^{i t s}-v(z)\right) e^{-i s z} / F^{\prime}(z) F^{*}(z)$ is bounded on every half-line $z=e^{i \theta}$, where $0 \leqslant \theta \leqslant \frac{1}{8} \pi$ or $\frac{7}{8} \pi \leqslant \theta \leqslant \pi$. Applying the Phragmén-Lindelöf theorem ([14], Chapter V, § 5.6), we conclude that the function in question is bounded in the upper half-plane. In other words, we have the relation

$$
\left|\frac{\left(e^{i t z}-v(z)\right) e^{-i s s}}{F(z) F^{*}(z) \mid}\right|=O\left(|z|^{2}\right) \quad(t>0, s<0)
$$

in the upper half-plane. Hence, in view of Cauchy's theorem, we get

$$
\begin{aligned}
\int_{-\infty}^{\infty}\left(e^{i t u}-v(u)\right) e^{-i s u} g_{x}(u) d u & =0 \int_{-\infty}^{\infty} \frac{\left(e^{i t u}-v(u)\right) e^{-i s u}}{F(u) F^{*}(u)} d u \\
& =-\lim _{a \rightarrow \infty} e \int_{S_{0}} \frac{\left(e^{i t s}-v(z)\right) e^{-i s z}}{F(z) F^{*}(z)} d z=0,
\end{aligned}
$$

where $s_{e}$ denotes the semi-circle $z=e e^{i \theta}(0 \leqslant \theta \leqslant \pi)$. Equality (12) is thus proved. From (10) and (11) it follows that the process $x(t)$ is of Markovian character. Moreover, the prediction $\pi_{0} x(t) \quad(t>0)$ depends upon infinitely many derivatives $x(0), x^{\prime}(0), \ldots$

The above-mentioned examples show that processes of Markovian character may have different analytical structure. Therefore a complete analytical characterization of such processes seems to be extremely difficult. In the next section we shall give a representation theorem for a subclass of the class of processes of Markovian character.

\section{A representation theorem}

The inner product $(\xi, \eta)$ on $\mathfrak{H}_{x}$ induces a bilinear functional on the space $\mathscr{D}$ of all infinitely differentiable functions whose supports are compact. Namely, for every pair $\psi_{1}, \psi_{2}$ of functions from $\mathscr{D}$ we put

$$
\left(\psi_{1}, \psi_{2}\right)_{\mathscr{Q}}=\left(X\left(\psi_{1}\right), X\left(\psi_{2}\right)\right) .
$$

A generalized process $X$ is said to be distributionally complete if the convergence $\lim _{n \rightarrow \infty}\left(\psi_{n}, \psi_{n}\right)_{D}=0 \quad\left(\psi_{n} \in \mathscr{D}\right)$ implies the convergence to 0 of the sequence $\psi_{1}, \psi_{2}, \ldots$ in the sense of the space $\mathscr{D}^{\prime}$ of Schwartz distributions, i. e. the convergence

$$
\lim _{n \rightarrow \infty} \int_{-\infty}^{\infty} \psi_{n}(t) \varphi(t) d t=0
$$

for every $\varphi$ from $\mathscr{D}$. For distributionally complete processes the bilinear functional $\left(\psi_{1}, \psi_{2}\right)_{\mathscr{D}}$ is an inner product on $\mathscr{D}$. Moreover, the completion of $\mathscr{D}$ to a Hilbert space is a subset $\mathscr{D}_{X}^{\prime}$ of the space $\mathscr{D}^{\prime}$ of Schwartz distributions. The strong convergence in $\mathscr{D}_{X}^{\prime}$ implies the convergence in the sense of the theory of distributions. It is obvious that the Hilbert spaces $\mathfrak{S}_{X}$ and $\mathscr{D}_{X}^{\prime}$ are isomorphic. The extension of the mapping $X(\varphi) \rightarrow \varphi$ $(\varphi \in \mathscr{D})$ is a natural isomorphism $\pi$ between $\mathfrak{H}_{X}$ and $\mathscr{D}_{X}^{\prime}$.

Let $g_{X}(t)$ be the density of the absolutely continuous component of the spectral measure $\mu_{x}$. A sufficient condition that the stationary process $X$ be distributionally complete is that for every $\varphi$ from $\mathscr{D}$ the integral

$$
\int_{-\infty}^{\infty} \frac{|\hat{\varphi}(t)|^{2}}{g_{X}(t)} d t
$$

be finite. In fact, applying Parseval's equality and the Schwarz inequality, we have

$$
\begin{gathered}
\left|\int_{-\infty}^{\infty} \psi_{n}(t) \varphi(t) d t\right|^{2}=\left|\frac{1}{2 \pi} \int_{-\infty}^{\infty} \hat{\psi}_{n}(t) \hat{\varphi}(t) d t\right|^{2} \\
=\left|\frac{1}{2 \pi} \int_{-\infty}^{\infty} \hat{\psi}_{n}(t) \sqrt{g_{X}(t)} \frac{\hat{\varphi}(t)}{\sqrt{g_{X}(t)}} d t\right|^{2} \leqslant \frac{1}{4 \pi^{2}} \int_{-\infty}^{\infty}\left|\hat{\psi}_{n}(t)\right|^{2} g_{X}(t) d t \int_{-\infty}^{\infty} \frac{|\hat{\varphi}(t)|^{2}}{g_{X}(t)} d t \\
\leqslant \frac{1}{4 \pi^{2}} \int_{-\infty}^{\infty}\left|\hat{\psi}_{n}(t)\right|^{2} \mu_{X}(d t) \int_{-\infty}^{\infty} \frac{\left.|| \hat{\varphi}(t)\right|^{2}}{g_{X}(t)} d t=\left(\psi_{n}, \psi_{n}\right)_{\mathscr{I}} \int_{-\infty}^{\infty} \frac{|\hat{\varphi}(t)|^{2}}{g_{X}(t)} d t
\end{gathered}
$$

which gives the distributional completeness. For instance, the integral

$$
\int_{-\infty}^{\infty} \frac{\left.\hat{\varphi}(t)\right|^{2}}{g_{X}(t)} d t
$$

is finite whenever $g_{X}$ is a positive polynomial or the reciprocal of a polynomial. Thus generalized processes with orthogonal values whose spectral density is a positive polynomial and ordinary processes whose spectral density is the reciprocal of a polynomial are distributionally complete. We have seen that processes belonging to these two classes are of Markovian character. The converse implication is also true. Namely, we shall prove the following theorem, where two processes $X$ and $Y$ are said to be equivalent if for every function $\varphi$ from $\mathscr{D}$ the equality $X(\varphi)=Y(\varphi)$ holds with probability 1 :

THEOREM 1. Every generalized stationary and distributionally complete process of Markovian character is equivalent to either a generalized proeess 
with orthogonal values whose spectral density is a positive polynomial or. an ordinary process whose spectral density is the reciprocal of a polynomial.

Before proving the Theorem we shall prove two Lemmas.

LEMMA 1. If the covariance function of a process $x(t) n$ times aifferentiable in quadratic mean is given on the right half-line by the formula

$$
r_{x}(t)=\sum_{k=1}^{m} v_{k}(t) \exp \left(a_{k} t\right)
$$

where $a_{1}, a_{2}, \ldots, a_{m}$ are different complex numbers, $v_{1}(t), v_{2}(t), \ldots, v_{m}(t)$ $\left(v_{k}(t) \not \equiv\right)$ are polynomials of degree $s_{1}, s_{2}, \ldots, s_{m}$ respectively and

$$
m+\sum_{k=1}^{m} s_{k} \leqslant n+1,
$$

then either $x(t)$ is a random oscillation or the spectral density - of $x(t)$ is the reciprocal of a polynomial.

Proof. Since the covariance function is bounded on the whole line, the real parts of $a_{1}, a_{2}, \ldots, a_{m}$ are non-positive. Moreover, if $\operatorname{Re} a_{k}=0$, then the corresponding polynomial $v_{k}(t)$ is constant. Thus, for positive $t$, the covariance function $r_{x}(t)$ can be written in the form

$$
r_{x}(z)=\sum_{k=1}^{p} w_{k}(t) \exp \left(b_{k} t\right)+\sum_{k=1}^{q} A_{k} \exp \left(i c_{k} t\right),
$$

where $b_{1}, b_{2}, \ldots, b_{p}$ are complex numbers with negative real parts, $w_{1}(t), w_{2}(t), \ldots, w_{p}(t)$ polynomials of degree $p_{1}, p_{2}, \ldots, p_{p}$ respectively $c_{1}, c_{2}, \ldots, c_{q}$ are real numbers and $A_{1}, A_{2}, \ldots, A_{q}$ are constant coefficients. For $p=0$ or $q=0$ the corresponding expressions are assumed to be 0 . Consequently, inequality (14) can be written in the form

$$
p+q+\sum_{k=1}^{\lfloor p} p_{k} \leqslant n+1 .
$$

Further, from the equality

$$
\mu_{x}\left(\left\{c_{k}\right\}\right)=\lim _{T \rightarrow \infty} \frac{1}{T} \int_{0}^{T} r_{x}(t) \exp \left(-i c_{k} t\right) d t=A_{k}
$$

it follows that all coefficients $A_{1}, A_{2}, \ldots, A_{q}$ are positive. Consequently, $r_{x}(t)$ is the covariance function of a random oscillation, provided $p=0$ Now let us suppose that $p \geqslant 1$. It is very easy to verify that the density $g_{x}$ of the absolutely continuous component of the spectral measure $\mu_{x}$ is given by the formula

$$
\left.g_{x}(t)=\frac{1}{\pi} \int_{0}^{\infty} \operatorname{Re}\left(\sum_{k=1}^{p} w_{k}(u) \exp \left(\left(b_{k}-i t\right) u\right)\right)\right) d u .
$$

Hence, by simple computations, we infer that $g_{x}(t)$ is a rational funetion: $g_{x}(t)=V(t) / W(t)$, where the polynomials $V(t)$ and $W(t)$ are supposed to have no common roots. Denoting by $d_{1}$ and $d_{2}$ the degree of $\nabla(t)$ and $W(t)$ respectively, we obtain, by virtue of (16), the inequality

$$
d_{2} \leqslant 2\left(p+\sum_{k=1}^{p} p_{k}\right)
$$

By assumption the process $x(t)$ is $n$ times differentiable in quadratic mean, whence the finiteness of the integral $\int_{-\infty}^{\infty} t^{2 n} \mu_{x}(d t)$ follows (see [2], p. 537). Consequently, the integral

$$
\int_{-\infty}^{\infty} t^{2 n} \frac{\nabla(t)}{W(t)} d t
$$

is also finite, which shows that the degree of $W(t)$ should be greater than the degree of $t^{2 n+1} V(t)$, i. e.

$$
2 n+2+d_{1} \leqslant d_{2}
$$

Hence, in view of (15) and (17) we obtain

$$
2 n+2+d_{1} \leqslant 2\left(p+\sum_{k=1}^{p} p_{k}\right) \leqslant 2\left(p+q+\sum_{k=1}^{p} p_{k}\right) \leqslant 2 n+2 .
$$

Consequently, $d_{1}=0$ and $q=0$, which implies that the spectral measure $\mu_{x}$ is absolutely continuous and its spectral density is the reciprocal of a polynomial. The lemma is thus proved.

LEMTMa 2. Let $x(t)$ be an ordinary stationary process. If there exist a positive number $t_{*}$ and a system of functions $a_{0}(t), a_{1}(t), \ldots, a_{n}(t)$ defined on the interval $0 \leqslant t<t_{*}$ such that for all $t$ from this interval the prediction $\pi_{0} x(t)$ is given by the formula

$$
\pi_{0} x(t)=\sum_{k=0}^{n} a_{k}(t) x^{(k)}(0),
$$

then either $x(t)$ is a random oscillation or the spectral density of $x(t)$ is the reciprocal of a polynomial.

Proof. From (18) for every positive number $u$ and every number $t$ satisfying the condition $0 \leqslant t<t_{*}$ we get

$$
\begin{aligned}
r_{x}(t+u)=(x(t), x(-u))=\left(\pi_{0} x(t), x(-u)\right) & \\
= & \sum_{k=0}^{n} a_{k}(t)\left(x^{(k)}(0), x(-u)\right)=\sum_{k=0}^{n} a_{k}(t) r_{x}^{(k)}(u) .
\end{aligned}
$$


If all the functions $a_{0}(t), a_{1}(t), \ldots, a_{n}(t)$ are identically equal to 0 then, by the continuity of the covariance function, $r_{x}(0)=0$ and, consequently, the process $x(t)$ is equivalent to a constant one and, of course, is a trivial random oseillation. Therefore, in the sequel we may suppose that the covariance function $r_{x}(t)$ and the function $a_{n}(t)$ are not identically equal to 0 .

First let us suppose that $n=0$. From the continuity of $r_{x}(t)$ we obtain, in view of (19), the continuity of $a_{0}(t)$ in the interval $0 \leqslant t \leqslant t_{*}$. Taking a positive number $u_{0}$ for which $r_{x}\left(u_{0}\right) \neq 0$ for every pair $t_{1}, t_{2}$ of positive numbers satisfying the condition $0 \leqslant t_{1}+t_{2}<t_{*}$ we have, according to (19), the equality

$$
a_{0}\left(t_{1}+t_{2}\right) r_{x}\left(u_{0}\right)=r_{x}\left(t_{1}+t_{2}+u_{0}\right)=a_{0}\left(t_{1}\right) r_{x}\left(t_{2}+u_{0}\right)=a_{0}\left(t_{1}\right) a_{0}\left(t_{2}\right) r_{x}\left(u_{0}\right),
$$

whence the equation $a_{0}\left(t_{1}+t_{2}\right)=a_{0}\left(t_{1}\right) a_{0}\left(t_{2}\right)$ follows. It is well known that the exponential functions $e^{-c t}$ are the only non-trivial solutions of this equation. Thus, for all positive $u$ and $t$ from the interval $0 \leqslant t<t$, we have the equality $r_{x}(t+u)=e^{-c t} r_{x}(u)$, which leads to the differential equation $r_{x}^{\prime}(u)=-c r_{x}(u)(u>0)$. Hence we infer that $r_{x}(t)$ is the covariance function of a stationary Markov process in the wide sense ([2], p. 233), which, of course, satisfies the assertion of the Lemma

Now let us suppose that $n \geqslant 1$. Taking a point $t_{0}$ for which $a_{n}\left(t_{0}\right)$ $\neq 0$ we infer, in view of (19), that for all positive $u$ the $n$-th derivative $r_{x}^{(n)}(u)$ is a linear combination of differentiable functions $r_{x}\left(u+t_{0}\right), r_{x}(u)$, $r_{x}^{\prime}(u), \ldots, r_{x}^{(n-1)}(u)$. Consequently, the covariance function $r_{x}(t)$ is infinitely differentiable on the right half-line. Now we shall prove that it satisfies on the right half-line a linear differential equation with constant coefficients:

$$
r_{x}^{(\boldsymbol{q}+1)}(t)+\sum_{k=0}^{q} c_{k} r_{x}^{(k)}(t)=0
$$

where

$$
q \leqslant n
$$

Let us introduce the notation

$$
D_{s}\left(t_{0}, t_{1}, \ldots, t_{s}\right)=\left|\begin{array}{cccc}
r_{x}\left(t_{0}\right) & r_{x}^{\prime}\left(t_{0}\right) & \ldots & r_{x}^{(s)}\left(t_{0}\right) \\
r_{x}\left(t_{1}\right) & r_{x}^{\prime}\left(t_{1}\right) & \ldots & r_{x}^{(s)}\left(t_{1}\right) \\
\ldots & \ldots & \ldots & \ldots \\
r_{x}\left(t_{s}\right) & r_{x}^{\prime}\left(t_{s}\right) & \ldots & r_{x}^{(s)}\left(t_{s}\right)
\end{array}\right|
$$

Of course, $D_{0}\left(t_{0}\right) \not \equiv 0$ on the right half-line. Now we distinguish two eases. First let us assume that $D_{n}\left(t_{0}, t_{1}, \ldots, t_{n}\right)=0$ for every system of positive numbers $t_{0}, t_{1}, \ldots, t_{n}$. Let $q$ be the least integer for which
$D_{q}\left(t_{0}, t_{1}, \ldots, t_{q}\right) \not \equiv 0$ and $D_{q+1}\left(t_{0}, t_{1}, \ldots, t_{q+1}\right) \equiv 0$. Obviously, $q<n$. Take a system $\bar{t}_{0}, \bar{t}_{1}, \ldots, \bar{t}_{q}$ satisfying the condition $D_{q}\left(\bar{t}_{0}, \bar{t}_{1}, \ldots, \bar{t}_{q}\right) \neq \mathbf{0}$. By developing the determinant $D_{q+1}\left(\bar{t}_{0}, \bar{t}_{1}, \ldots, \bar{t}_{q}, t\right)$ with respect to the last row, we get

$$
\frac{D_{q+1}\left(\bar{t}_{0}, \bar{t}_{1}, \ldots, \bar{t}_{q}, t\right)}{D_{q}\left(\bar{t}_{0}, \bar{t}_{1}, \ldots, \bar{t}_{q}\right)}=r_{x}^{(q+1)}(t)+\sum_{k=0}^{k k} c_{k} r_{x}^{(k)}(t)=0 \quad(t>0),
$$

where the coefficients $c_{0}, c_{1}, \ldots, c_{q}$ do not depend upon $t$. Relations (20) and (21) are thus proved.

Finally let us suppose that $D_{n}\left(\bar{t}_{0}, \bar{t}_{1}, \ldots, \bar{t}_{n}\right) \neq 0$ for a system $\bar{t}_{0}, \bar{t}_{1}$, $\ldots, \bar{t}_{n}$ of positive numbers. Introducing the notation

$$
\Delta_{h}^{(s)} f(u)=\sum_{k=0}^{s}(-1)^{k}\left(\begin{array}{l}
s \\
k
\end{array}\right) f(u+(s-k) h)
$$

we get, in view of (19), the equation

$$
\Delta_{h}^{(n+1)} \cdot r_{x}(u)=\sum_{k=0}^{n} r_{x}^{(k)}(u) \Delta_{h}^{(n+1)} a_{k}(0)
$$

whenever $u>0$ and $0 \leqslant(n+1) h<t_{*}$. Substituting $u=\bar{t}_{0}, \bar{t}_{1}, \ldots, \bar{t}_{n}$ into the last equation, we get a system of linear equations for quantities $\Delta_{h}^{(n+1)} a_{k}(0)(k=0,1, \ldots, n)$. The determinant of this system, being equal to $D_{n}\left(\bar{t}_{0}, \bar{t}_{1}, \ldots, \bar{t}_{n}\right)$, is different from 0 . Thus $\Delta_{h}^{(n+1)} a_{k}(0)(k=0,1, \ldots, n)$ can be expressed as linear combinations of $\Delta_{h}^{(n+1)} r_{x}\left(t_{j}\right)(j=0,1, \ldots, n)$ whose coefficients do not depend upon $h$. Hence we obtain the convergence

$$
\frac{1}{h^{n+1}} \Delta_{h}^{(n+1)} a_{k}(0) \rightarrow-c_{k} \quad(k=0,1, \ldots, n)
$$

as $h \rightarrow 0$. Dividing by $h^{n+1}$ equality (22) we get, as $h \rightarrow 0$, the equation $r_{x}^{(n+1)}(u)+\sum_{k=0}^{n} \theta_{k} r_{x}^{(k)}(u)=0 \quad(u>0)$, which completes the proof of $(20)$ and (21).

From equation (20) it follows that for positive $t$ the covariance function $r_{x}(t)$ is of the form $\sum_{k=1}^{m} v_{k}(t) \exp \left(a_{k} t\right)$, where $a_{1}, a_{2}, \ldots, a_{m}$ are different complex numbers and $v_{1}(t), v_{2}(t), \ldots, v_{m}(t)$ are polynomials not identically equal to 0 . Denoting by $s_{1}, s_{2}, \ldots, s_{m}$ their degrees, we have, according to (21), the inequality

$$
m+\sum_{k=1}^{m} s_{k} \leqslant n+1
$$

Further, we have supposed that $a_{n}(t) \neq 0$ in the interval $0 \leqslant t<t_{*}$. Consequently, by formula (18), the process $x(t)$ is $n$ times differentiable. Applying Lemma 1 we get the assertion of our Lemma. 
Proof of Theorem 1. Suppose that a generalized process $X$ satisfies the assumptions of the Theorem. Taking a function $p$, whose support is contained in the right half-line, we have the relation $\pi_{0} X(p) \in \bigcap \mathfrak{H}_{X}^{(u, 0)}$. Consequently, we can find a sequence $\psi_{1}, \psi_{2}, \ldots$ of functions from in such a way that

$$
s\left(\psi_{n}\right) \subset\left(-\frac{1}{n}, 0\right) \quad(n=1,2, \ldots)
$$

and the sequence $X\left(\psi_{1}\right), X\left(\psi_{2}\right), \ldots$ converges to $\pi_{0} X(\varphi)$ in quadratic mean. In the language of the space $L^{2}\left(\mu_{X}\right)$ this means that the sequence of Fourier transforms $\hat{\psi}_{1}, \hat{\psi}_{2}, \ldots$ converges to a function $f$ from $L^{2}\left(\mu_{X}\right)$ in the sense of $L^{2}\left(\mu_{X}\right)$-convergence and

$$
\iota\left(\pi_{0} X(\varphi)\right)=f,
$$

where $\iota$ denotes the natural isomorphism between $\mathfrak{H}_{X}$ and $L^{2}\left(\mu_{X}\right)$. Moreover, from the distributional completeness of $X$ it follows that the sequence $\psi_{1}, \psi_{2}, \ldots$ is distributionally convergent to a distribution $T$ from $\mathscr{D}_{X}^{\prime}$. By formula (23), the distribution $T$ is concentrated at the origin. Consequently, $T$ is a linear combination of the Dirac $\delta$-distribution and its derivatives

$$
T=\sum_{k=0}^{m} b_{k} \delta^{(k)}
$$

where, of course, the coefficients $b_{0}, b_{1}, \ldots, b_{m}$ and the integer $m$ depend upon $\varphi$ (see [13], Chapter III, § 10).

First let us suppose that for every function $\varphi$ from $\mathscr{D}$ whose support is contained in $(0, \infty)$ the corresponding distribution $T$ vanishes. Hence we get the equality $\pi_{0} X(\varphi)=0$ whenever $s(\varphi) \subset(0, \infty)$. Consequently, the process $X$ has orthogonal values.

Now we shall prove that the spectral density of $X$ is a positive polynomial. Contrary to this let us suppose that the spectral density of $X$ is a polynomial having a real root. Since this polynomial is non-negative, its every real root must be of even multiplicity. Consequently, the spectral density $g_{X}$ may be written in the form $g_{X}(t)=(t-a)^{2} v(t)$, where $a$ is a real number and $v(t)$ is a polynomial. We define auxiliary functions $f_{1}, f_{2}, \ldots, f_{\infty}$ setting

$$
\begin{aligned}
& f_{k}(t)=\left\{\begin{array}{cc}
\frac{1}{t-a} & \text { if } \quad a+\frac{1}{k}<t, \quad(k=1,2, \ldots) \\
0 & \text { otherwise, }
\end{array}\right. \\
& f_{\infty}(t)=\left\{\begin{array}{cl}
\frac{1}{t-a} & \text { if } a<t, \\
0 & \text { otherwise. }
\end{array}\right.
\end{aligned}
$$

Obviously, all the functions $f_{1}, f_{2}, \ldots, f_{\infty}$ belong to $L^{2}\left(\mu_{X}\right)$ and the sequence $f_{1}, f_{2}, \ldots$ converges to $f_{\infty}$ in the sense of $L^{2}\left(\mu_{X}\right)$-convergence. Further, for every index $k$ we ean find a sequence $\psi_{1, k}, \psi_{2, k}, \ldots$ of functions from $\mathscr{D}$ such that

(26) $\quad \lim _{n \rightarrow \infty} \int_{-\infty}^{\infty}\left|\hat{\psi}_{n, k}(t)-f_{k}(t)\right|^{2}\left(1+g_{X}(t)\right) d t=0 \quad(k=1,2, \ldots)$.

Hence, in particular, it follows that the sequence $\hat{\psi}_{1, k}, \hat{\psi}_{2, k}, \ldots$ is convergent to $f_{k}$ in the sense of $L^{2}\left(\mu_{X}\right)$-convergence and, consequently, by the distributional completeness of the process $X$, the sequence $\psi_{1, k}$, $\psi_{2, k}, \ldots$ is distributionally convergent to a distribution, say $T_{k}$, from $\mathscr{D}_{X}^{\prime}$. Moreover, the sequence $T_{1}, T_{2}, \ldots$ of distributions is distributionally convergent in $\mathscr{D}_{X}^{\prime}$. In order to obtain an explicit formula for $T_{k}$ we apply the Schwarz inequality and Parseral's equality:

$$
\begin{aligned}
& \left|\int_{-\infty}^{\infty} \psi_{n, k}(t) \varphi(t) d t-\frac{1}{2 \pi} \int_{1 / k}^{\infty} \frac{\hat{\varphi}(t+a)}{t} d t\right|^{2} \\
& =\left|\frac{1}{2 \pi} \int_{-\infty}^{\infty} \hat{\psi}_{n, k}(t) \hat{\varphi}(t) d t-\frac{1}{2 \pi} \int_{-\infty}^{\infty} f_{k}(t) \hat{\varphi}(t) d t\right|^{2} \\
& \leqslant \frac{1}{4 \pi^{2}}\left(\int_{-\infty}^{\infty}\left(1+g_{X}(t)\right)\left|\hat{\psi}_{n, k}(t)-f_{k}(t)\right||\hat{\varphi}(t)| \frac{d t}{1+g_{X}(t)}\right)^{2} \\
& \leqslant \frac{1}{4 \pi^{2}}\left(\int_{-\infty}^{\infty} \frac{d t}{1+g_{X}(t)}\right)^{2} \int_{-\infty}^{\infty}\left(1+g_{X}(t)|| \hat{\psi}_{n, k}(t)-\left.f_{k}(t)\right|^{2} d t \int_{-\infty}^{\infty} \frac{|\hat{\varphi}(t)|^{2}}{1+g_{X}(t)} d t .\right.
\end{aligned}
$$

The integral

$$
\int_{-\infty}^{\infty} \frac{|\hat{\varphi}(t)|^{2}}{1+g_{X}(t)} d t
$$

is finite for all functions $\varphi$ from $\mathscr{D}$. Further, the integral

$$
\int_{-\infty}^{\infty} \frac{d t}{1+g_{X}(t)}
$$

is finite, because $1+g_{X}(t)$ is a positive polynomial of degree at least 2 . Consequently, according to (26), we get the formula

$$
T_{k}(\varphi)=\frac{1}{2 \pi} \int_{1 / k}^{\infty} \frac{\hat{\varphi}(t+a)}{t} d t=\frac{\hat{\varphi}(a)}{2 \pi} \log k+O(1) \quad(k=1,2, \ldots)
$$


which contradicts the distributional convergence of $T_{1}, T_{2}, \ldots$ Thus $g_{X}$ is a positive polynomial.

Now let us suppose that there exists a function $\varphi$ belonging to $\mathscr{D}$ for which the distribution $T$ defined by formula (25) is different from 0 . Let $\psi_{1}, \psi_{2}, \ldots$ be the sequence defined above and eonvergent to the distribution $T$ in the distributional sense. The supports of these functions satisfy condition (23) and the sequence of their Fourier transforms converges to a function $f$ from $L^{2}\left(\mu_{X}\right)$ satisfying equality (24). From the distributional convergence of the sequence $\psi_{1}, \psi_{2}, \ldots$ it follows that there exist an integer $s$ and a sequence $F_{1}, F_{2}, \ldots$ of continuous functions which is uniformly convergent to a continuous function $F$ in every finite interval and

$$
\begin{gathered}
F_{n}^{(s)}=\psi_{n} \quad(n=1,2, \ldots), \\
F^{(s)}=T,
\end{gathered}
$$

where the last derivative is taken in the distributional sense ([13], Chapter III, §6). From (27) we get the equality

$$
\Delta_{h}^{(s)} F_{n}(t)=\int_{t}^{t+h} \int_{t_{s}}^{t_{s}+h} \ldots \int_{t_{2}}^{t_{2}+h} \psi_{n}\left(t_{1}\right) d t_{1} d t_{2} \ldots d t_{s}
$$

which implies the following one:

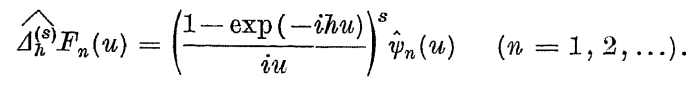

Hence it follows that the sequence $\widehat{\Delta_{h}^{(s)}} F_{1}(u), \widehat{\Delta_{h}^{(s)} F_{2}}(u), \ldots$ is convergent to the function

$$
\left(\frac{1-\exp (-i \hbar u)}{i u}\right)^{s} f(u)
$$

in the sense of $L^{2}\left(\mu_{X}\right)$-convergence. On the other hand, in view of (23) and (29), the supports of the functions $\Delta_{h}^{(s)} F_{n}(t)(n=1,2, \ldots)$ are bounded in common, which implies the uniform convergence to $\widehat{\Delta_{h}^{(s)}} F(u)$ of the sequence $\widehat{\Delta_{h}^{(s)} F_{1}}(u), \widehat{\Delta_{h}^{(s)} F_{2}}(u), \ldots$ Hence, for every real number $h$, we get the equality

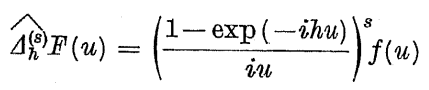

$\mu_{X}$-almost everywhere.

Further, according to (28), by simple computations we get the equation



where the polynomial $\sum_{k=0}^{m} b_{k}(-i u)^{k}$ is the generalized Fourier transform of the distribution $T$ (see [4], p. 212, [13], Chapter VII, §2). Comparing the last two equalities we get, for every real number $h$, the equality

$$
\left(\frac{1-\exp (-i h u)}{i u}\right)^{s} f(u)=\left(\frac{1-\exp (-i h u)}{i u}\right)^{s} \sum_{k=0}^{m} b_{k}(-i u)^{k}
$$

$\mu_{X}$-almost everywhere. Consequently, to every number $h$ there correspond a set $A_{h}$ of $\mu_{X}$-measure 0 and a set $B_{h}$ consisting of multiples of $2 \pi / h$ different from 0 such that the equality

$$
f(u)=\sum_{k=0}^{m} b_{k}(-i u)^{k}
$$

holds whenever $u \in A_{h} \cup B_{h}$. Taking a pair $h_{1}, h_{2}$ of incommeasurable numbers we observe that $B_{h_{1}} \cap B_{h_{2}}=0$ and, furthermore, formula (30) holds if $u$ do not belong to the intersection $\left(A_{h_{1}} \cup B_{h_{1}}\right) \frown\left(A_{h_{2}} \cup B_{h_{2}}\right)$, which is contained in $A_{h_{1}} \cup A_{h_{2}}$. Consequently, equality (30) holds $\mu_{X^{-}}$ -almost everywhere. In other words, the polynomial $\sum_{k=0}^{m} b_{k}(-i u)_{k}$ belongs to $L^{2}\left(\mu_{X}\right)$. Of course, we may assume that $b_{m} \neq 0$, which implies the inequality $\int_{-\infty}^{\infty} u^{2 m} \mu_{X}(d u)<\infty$. Hence it follows that the process $X$ is equivalent to an ordinary process $x(t) m$ times differentiable (see [2], p. 537). In the same way as previously in the proof of formula (25) we can prove that for every positive number $t$ there exists a linear combination $T_{t}=\sum_{k=0}^{n_{t}}(-1)^{k} a_{k}(t) \delta^{(k)}$ such that, according to $(24)$ and $(30)$

$$
\iota\left(\pi_{0} x(t)\right)=\sum_{k=0}^{n_{t}} a_{k}(t)(i \cdot u)^{k}
$$

holds. Hence we get the equality

$$
\pi_{0} x(t)=\sum_{k=0}^{n_{t}} a_{k}(t) x^{(k)}(0) \quad(t \geqslant 0) .
$$

By continuity in quadratic mean of the prediction $\pi_{0} x(t)$ with respect to $t$, the orders of the distributions $T_{t}$ are bounded in common when runs over a finite interval. Consequently, for every positive number $t_{*}$ we can find a number $n$ such that for all $t$ satisfying the inequality $0 \leqslant t \leqslant t_{*}$ the quantity $n_{t}$ from formula (31) is equal to $n$. Applying Lemma 2 , we conclude that either the spectral density of $x(t)$ is the reciprocal of a polynomial or $x(t)$ is a random oscillation. Consequently, 
to prove the Theorem it suffices to show that the random oscillations are not distributionally complete.

Consider a random oscillation $x(t)$ with the covariance function of form (2). We can easily find a function $\varphi_{0}\left(\varphi_{0} \neq 0\right)$ belonging to $\mathscr{D}$ whose Fourier transform vanishes at the points $\lambda_{1}, \lambda_{2}, \ldots, \lambda_{n}$. From the equality $\left(\varphi_{0}, \varphi_{0}\right)_{\mathscr{D}}=\int_{-\infty}^{\infty}\left|\hat{\varphi}_{0}(t)\right|^{2} \mu_{x}(d t)=\sum_{k=1}^{n} b_{k}\left|\hat{\varphi}_{0}\left(\lambda_{k}\right)\right|^{2}=0$ it follows that the bilinear functional $(\varphi, \psi)_{\mathscr{D}}$ is not an inner product on $\mathscr{D}$ and, consequently, the random oscillation $x(t)$ is not distributionally complete. The theorem is thus proved.

\section{Strong Markovian character}

A generalized stationary process $X$ is said to be of strong Markovian character if for every generalized stationary process $Y$ orthogonal to $X$ and for every function $\varphi$ from $\mathscr{D}$ whose support is contained in the right half-line the prediction $\pi_{0}^{X+{ }^{Y}} X(\varphi)$ of $X(\varphi)$ based on the full past of the process $X+Y$ up to time 0 belongs to $\bigcap_{u<0} \mathfrak{S}_{X+Y}^{(u, 0)}$. A complete characterization of processes of strong Markovian character is given by the following theorem, which is a consequence of Theorem 1:

THEOREM 2. Generalized stationary processes with orthogonal values are the only processes of strong Markovian character.

First of all we shall prove two simple Lemmas.

LEMMA 3. The sum of two orthogonal processes of strong Markovian character is of Markovian eharacter.

Proof. Given two orthogonal processes $X$ and $Y$ of strong Markovian character, we have the relations

$$
\pi_{0}^{X+P} X(\varphi) \in \bigcap_{u<0} \mathfrak{H}_{X^{+}+F}^{(u, 0)}, \quad \pi_{0}^{X+F} X(\varphi) \in \bigcap_{u<0} \mathfrak{H}_{X+Y}^{(u, 0)},
$$

whenever $s(\varphi) \subseteq(0, \infty)$. Hence, by equality

$$
\pi_{0}^{X+Y}(X(\varphi)+Y(\varphi))=\pi_{0}^{X+Y} X(\varphi)+\pi_{0}^{X+Y} Y(\varphi),
$$

we get the relation

$$
\pi_{0}^{X+Y}(X(\varphi)+Y(\varphi)) \in \bigcap_{u<0} \mathfrak{S}_{X^{+}+Y}^{(u, 0)},
$$

which implies the Markovian character of the sum $X+Y$.

LeMcma 4. Let $X, Y$ be a pair of orthogonal processes. If the processes $Y$ and $X+Y$ have orthogonal values, then the process $X$ also has the orthogonal values.
Proof. Assuming that $s(\varphi) \frown s(\psi)=0(\varphi, \psi \in \mathscr{D})$, we have

$$
\begin{aligned}
(X(\varphi), X(\psi))= & (X(\varphi)+Y(\varphi), X(\psi)+Y(\psi))-(X(\varphi), Y(\psi))- \\
& -(Y(\varphi), X(\psi))-(Y(\varphi), Y(\psi))=0,
\end{aligned}
$$

which implies the assertion of the Lemma.

Proof of Theorem 2. First we shall prove that every generalized stationary process $X$ with orthogonal values is of strong Markovian character. Let $Y$ be an arbitrary stationary process orthogonal to $X$. Obviously, $X(\varphi)$ is orthogonal to $\mathfrak{S}_{X+Y}^{(-\infty, 0)}$ whenever $s(\varphi) \subset(0, \infty)$, which implies the strong Markovian character of the process $X$.

Now let us assume that a generalized stationary process $X$ is of strong Markovian character. Taking an arbitrary generalized stationary process $Y$ orthogonal to $X$ with orthogonal values with positive spectral density, we conclude, in view of Lemma 3 and the preceding part of the proof, that the sum $X+Y$ is of Markovian character. Further, the spectral density of $Y$, being a positive polynomial, is bounded below by a positive number. Consequently, the density $g_{X_{+} Y}$ of the absolutely continuous part of the spectral measure $\mu_{X+Y}$ is also bounded below by a positive number. Therefore, for every function $\varphi$ from $\mathscr{D}$, the integral

$$
\int_{-\infty}^{\infty} \frac{|\hat{\varphi}(t)|^{2}}{g_{X+Y}(t)} d t
$$

is finite. We have proved in Section III that the last condition implies distributional completeness. Thus the sum $X+Y$ is a distributionally complete process of Markovian character. By Theorem 1, the process $X+Y$ has orthogonal values, because its spectral density is bounded below by a positive number. Hence, and from Lemma 4 it follows that the process $X$ itself has orthogonal values, which completes the proof.

\section{References}

[1] Ю. К. Беляев, Аналитические случайные процессы, Теория вероятностей и ее применения IV (4) (1959), p. 437-444.

[2] J. L. Doob, Stochastic processes, New York-London 1953.

[3] И. М. Ге Ль фан д, Обобщенные случайные прочессы, Доклады Ак. Наук CGCP $100, N_{0} 5$ (1955), p. $853-856$.

[4] И. М. Г ель фанд Пи Г. Е. Шилов, Обобщенные функиии и действия над ними, Москва 1959.

[5] K. Itô, Stationary random distributions, Memoirs of the College of Science, [5] K. Itô, Stationary randity of Kyoto, Series A, 28, No 3 (1953), p. 209-223.

[6] А. М. Я тлом, Введение в теорию стачионарных случайных функұий, Успехи Математиqеских Наук VII (5) (1952), p. 3-168.

[7] А. Н. Колмогоров, Интерполирование и экстраполирование стаұионарных случайных последовательностей, Известия Акад. Наук СССР, сер. ма- тематич., 5 (1941), p. 3 -14. 
[8] М. Г. К рей н, Об одной экстраполяционной проблеме А. М. Колмогорова, [8]

[9]

[9] P. Lov $y, A$ special probin of Gaussian random functions, Proceedings of the This Bit thematical Statistics and Probability, Vol. II. Probability Theory, Berkeley-Lo Angeles 1956.

[10] Б. Я. Л е ви н, Pacn ределение порней цельх фуньиий, Москва 1956.

[11] [12] Теория вероятностей п ее грименения IV (4) (1959), p. 465-471.

процессов, Теория вероятностей й еe тitributions, I, II, Paris 1950-1951.

[13] L. Schwartz, Théorie des distributions, I, II, Paris 1950
[14] E. C. Titchmarsh, The theory of functions, Oxford 1932.

[15] N. Wiener, Extrapolation, interpolation and smoothing of stationary time series, Cambridge - New York 1949.

MATHEMATICAL INSTITUTE OF THE POLISH ACADEMY OF SCIENCES INSTYTUT MATEMATYCZNY POLSKIEJ AKADEMII NAUK

Reçu par la Rédaction le 16.6.1961

\section{On modified Landau polynomials}

by

\section{J. RADECKI (Poznań)}

This paper contains some theorems on the approximation of continuous functions $f(t)$ in an infinite interval by means of polynomials

$$
P_{n}[f(t) ; x]=\frac{\int_{0}^{1} f\left(h_{n} t\right)\left[1-\left(t-\frac{x}{h_{n}}\right)^{2}\right]^{n} d t}{2 \int_{0}^{1}\left(1-t^{2}\right)^{n} d t},
$$

where $h_{n}>0$ and $\lim h_{n}=\infty$. This kind of polynomials were first introduced by Hsu $[1,2]$, who also showed their convergence in the case of $h_{n}=n^{\Theta}$ and $f(t)$ of certain classes of continuous functions. The results given in the present paper are more general.

THEOREM 1. If $x>0$, then $\lim _{n \rightarrow \infty} P_{n}(1 ; x)=1$ if and only if

$$
\lim _{n \rightarrow \infty} \frac{h_{n}}{\sqrt{n}}=0 \text {. }
$$

Proof. First we prove the sufficiency. Easy transformations give

$$
P_{n}(1 ; x)-1=\frac{\int_{x \sqrt{n} / h_{n}}^{\sqrt{n}}\left(1-\frac{u^{2}}{n}\right)^{n} d u+\int_{\left(1-x / h_{n}\right) \sqrt{n}}^{\sqrt{n}}\left(1-\frac{u^{2}}{n}\right)^{n} d u}{2 \int_{0}^{\sqrt{n}}\left(1-\frac{u^{2}}{n}\right)^{n} d u} .
$$

Since $\left(1-u^{2} / n\right)^{n} \leqslant e^{-u^{2}}$ for $|u| \leqslant \sqrt{n}$, we have

$$
0 \leqslant \int_{x \sqrt{n} / h_{n}}^{\sqrt{n}}\left(1-\frac{u^{2}}{n}\right)^{n} d u \leqslant \int_{x \sqrt{n} ; / h_{n}}^{\sqrt[1]{n}} e^{-u^{2}} d u,
$$

